\title{
Corrigendum
}

\section{Diagnosing undernutrition children and adults: new French criteria. Why, for what and for whom? A joint statement of the French National Authority for Health and French Federation of Nutrition - Corrigendum}

Jacques Delarue, Jean-Claude Desport, Béatrice Dubern, Francisca Joly, Emmanuel Mas, Alexandre Pitard and Eric Fontaine HAS Working Group

DOI: https://doi.org/10.1017/S0007114521001471 (First published online 07 May 2021)

The authors apologise for an omission in the list of authors from the HAS Working Group. The original text reads:

HAS Working Group affiliations: Frédéric Costes (Clermont-Ferrand), Marie-Laure De Botton (Lille), Anne Galinier (Toulouse), François Goldwasser (Paris), Bernard Grunberg (Miramas), Régis Hankard (Tours), Martine Le-Noc-Soudani (Paris); Yves Morel (Saint-Egrève), Agathe Raynaud-Simon (Paris); Florence Rossi Pacini (Marseille), Marcel Ruetsch (Dessenheim), David Seguy (Lille), Jean-Fabien Zazzo (Clamart) (FNA Working Group).

The correct list of HAS Working Group authors is as follows:

HAS Working Group affiliations: Frédéric Costes (Clermont-Ferrand), Marie-Laure De Botton (Lille), Anne Galinier (Toulouse), François Goldwasser (Paris), Bernard Grunberg (Miramas), Régis Hankard (Tours), Martine Le-Noc-Soudani (Paris); Yves Morel (Saint-Egrève), Agathe Raynaud-Simon (Paris); Florence Rossi Pacini (Marseille), Marcel Ruetsch (Dessenheim), David Seguy (Lille), Marie-Paule Vasson (Clermont-Ferrand), Jean-Fabien Zazzo (Clamart) (FNA Working Group). 